\title{
Pet food safety: a shared concern
}

\author{
Robert L. Buchanan ${ }^{1 *}$, Robert C. Baker ${ }^{2}$, Adrian J. Charlton ${ }^{3}$, Jim E. Riviere $^{4}$ and Robert Standaert ${ }^{5}$ \\ ${ }^{1}$ Center for Food Safety and Security Systems, University of Maryland, 0119 Symons Hall, College Park, MD 20742, USA \\ ${ }^{2}$ MARS, Inc., Bangkok, Thailand \\ ${ }^{3} U K$ Food and Environment Research Agency, Sand Hutton, York, UK \\ ${ }^{4}$ Center for Chemical Toxicology Research and Pharmacokinetics, North Carolina State University, Raleigh, NC, USA \\ ${ }^{5}$ Biosciences Division, Oak Ridge National Laboratory, Oak Ridge, TN, USA
}

(Received 11 November 2010 - Revised 5 August 2011 - Accepted 9 August 2011)

\section{Abstract}

The safety of the food supply is a subject of intense interest to consumers, particularly as a result of large-scale outbreaks that involve hundreds and sometimes thousands of consumers. During the last decade, this concern about food safety has expanded to include the diets of companion animals as a result of several incidences of chemical toxicities and infectious disease transmission. This has led to increased research into the causes and controls for these hazards for both companion animals and their owners. The following summary provides an introduction to the issues, challenges and new tools being developed to ensure that commercial pet foods are both nutritious and safe.

\section{Key words: Salmonella enterica: Melamine: Mycotoxins: Microbiological safety: Chemical safety: Toxicology: Analytical methods}

\section{Pet food safety in the spotlight}

The nutritional adequacy and safety of foods are intertwined in the minds of consumers. Foods that are nutritiously sound but that are not trusted because of safety concerns will be rejected by pet owners, as will foods that are safe but are considered nutritionally inadequate. Thus, regardless of whether the focus is human or pet nutrition, the ability to manufacture and distribute safe products is a prerequisite for marketing a food product. This is particularly important for infants and pets, where the products are often the sole source of nutrition.

Companion animals are an integral part of the family unit in most societies. As the size of families has decreased in most developed countries, the role of pets as 'family members' has increased dramatically. The health and well-being of pets is a responsibility that most owners take very seriously. As a result, owners increasingly rely on high-quality, commercially prepared pet foods to provide both convenience and sound nutrition through the different phases of an animal's life. However, as consumers increasingly rely on commercially produced pet foods, the potential impact of hazards associated with the manufacture, distribution and use of pet foods is amplified as a result of common sourcing of ingredients and increased size of production lots. Thus, an error in manufacturing could lead to large-scale adverse events.
Pet food safety represents a substantial challenge over traditional food safety concerns because the hazards can potentially directly have an impact on the animal and indirectly affect humans who share its environment. Pet food can serve as a vehicle for infectious disease agents, as well as naturally occurring and inadvertently added toxicants. It can also potentially lead to nutritional deficiencies if formulated incorrectly. The direct impact of chemical contaminants in pet foods on animal health was dramatically demonstrated in 2007 when cases of kidney damage and failure in cats and dogs were reported in the $\mathrm{USA}^{(1,2)}$. Ultimately, the source of disease was traced back to the adulteration of protein sources with melamine and cyanuric acid. However, this is not the only example of chemical hazards being associated with pet food ingredients. For example, in 2005-6, there were more than 100 deaths of dogs in the USA, which were linked to the consumption of pet foods containing high levels of aflatoxins, resulting in a large recall of pet food ${ }^{(3)}$ and, again, emphasising the impact that improper ingredient sourcing can have on the safety of the product.

The impact that the microbiological contamination of pet foods has on animal health is less clear-cut due to the fact that few of the potential outbreaks are investigated thoroughly and are often confounded by the multiple routes of exposure. However, it is clear that many of the same pathogenic 
micro-organisms that affect humans also cause disease in companion animals. For example, improperly canned dog food has a similar risk of botulism due to toxin production by Clostridium botulinum as it has for human foods. Likewise, while adult dogs are generally less likely to be symptomatic than humans, Salmonella enterica infections in dogs are relatively common, and salmonellosis can be a serious disease in puppies and elderly animals ${ }^{(4-8)}$.

The ability of companion animals to serve as reservoirs for disease agents that can have an impact on their owners and other humans has long been recognised. For example, the common canine parasite Toxocara canis can also infect humans. The transmission of this nematode is associated with the contamination of the environment by faecal material. Another example is the warning given to pregnant women to avoid handling of feline faecal material, as well as avoiding handling and consuming undercooked meats, to reduce the risk of fetal Toxoplasma gondii infections. However, it has only recently become widely appreciated that pet foods can be a source of pathogenic micro-organisms that can have an impact on pet owners. Pet foods can serve as a vehicle for foodborne pathogens, resulting in direct or indirect transmission. In the former, the handling (or in some cases ingestion) of contaminated pet foods and treats by the pet owner is the route of transmission. In the latter case, the pet becomes asymptomatically infected, and, in turn, serves as a reservoir for the pathogenic micro-organism either through direct contact between the pet and the pet owner, or through faecal contamination of the environment. Several recent outbreaks of human salmonellosis have been directly linked to contamination of dry pet foods and pet treats (e.g. pig ears) ${ }^{(9,10)}$.

The safety of pet foods in many countries has traditionally been held to a higher standard than animal feed. Furthermore, the recent outbreaks associated with chemical or microbiological contamination of pet foods have resulted in several countries revisiting the requirements associated with manufacture and sale of these products. For example, the US Food and Drug Administration clearly articulated its expectations that pet food be manufactured under conditions similar to those that it requires for human food. A number of large recalls have been undertaken by manufacturers after dry pet foods and treats have been found to be contaminated with $S$. enterica or manufactured with ingredients that were subsequently found to be contaminated with the pathogen. For example, a substantial portion of the products recalled as a result of the widespread contamination of peanuts and peanut flours with $S$. enterica were pet foods. Other countries have similar concerns and changes in regulatory approaches are being implemented in Europe, Japan and other countries. These changes in consumer expectations and accompanying regulatory interest are likely to have major impacts on the pet food industry, particularly for dry pet food products. The most obvious will be a wide scale need to upgrade facilities so that they meet the requirements for the production of human foods. However, it will also have an impact on all operations as the need for more care in the acquisition of ingredients, the maintenance of records and the level of quality assurance will be expected by both retail markets and consumers.

\section{Refocusing microbiological safety of pet foods}

The recent, highly publicised salmonellosis outbreaks and recalls of dry pet foods due to contamination with $S$. enterica have caused a major review of microbiological control programmes, and have reinforced the idea that food safety goes beyond traditional factory quality management processes. As in foods for humans, ensuring the microbiological integrity of pet foods must cover the entire production pipeline ('farm-to-fork approach'). Failure to do so can place both your consumers and their animals, and thus your products and business, at risk of a food safety incident. Historical information demonstrates that food safety incidents can be traced back to issues involving raw materials, production, distribution and mishandling before consumption. Looking further into these food safety incidents, it is possible to categorise the root cause(s) as chemical (involving contamination with toxic chemicals that are added directly or indirectly), physical (involving contamination with foreign objects) and microbiological (contamination with hazardous micro-organisms and/ or their toxic metabolites). Of these categories, food safety issues of microbiological origin have been the most challenging for the food industry and have received increasing focus over the past 5 years. This effort has escalated with recent advances in forensic epidemiology, which has increased our ability to detect diffuse outbreaks on regional, national and even international levels. During the past 5 years, there have been numerous food recalls due to microbiological issues related to mycotoxins (primarily aflatoxin) and S. enterica contamination. The mycotoxin issues are typically traced back to the usage of contaminated raw materials (typically grains), which are not detected before the food manufacturing process. The Salmonella issues are not that straightforward and, in several recent incidents, have involved food types that have been traditionally viewed as low risk (i.e. high-acid or low-water-activity foods). To highlight this, during the past 2 years, there have been Salmonella outbreaks associated with tomato-based salsa (high-acid food), peanuts (low-water-activity food), chicken pot pies (frozen food) and dry pet food (low-water-activity food). Past beliefs were that Salmonella would not survive in or on these foods, and would therefore be low risk of a food safety incident. However, Salmonella (and a number of other enteric bacteria) have survival mechanisms that allow it to survive in dry products, sometimes for extended periods (up to a year or longer). In addition, it has repeatedly been implicated in outbreaks where contamination rates were $\leq 1 \mathrm{cell} / \mathrm{g}$. Recent outbreaks have shown that Salmonella can survive on dry pet foods, and serve as a vehicle of contamination and infection to both the pet and the pet owners. This is primarily driven by the fact that, in many instances, pets and their food are in the home. Thus, it is critical that pet foods are produced in a manner that prevents Salmonella contamination.

Managing Salmonella contamination in the production of dry pet foods can be very challenging as many of the raw 
materials are naturally contaminated (i.e. grains, meats and meat meals, poultry, etc.). Therefore, it is crucial to perform a risk assessment to understand which materials and processes pose the most risk and develop a control plan that addresses and minimises the potential product safety concerns. This must be comprehensive and take into account key risk areas and risk management efforts involving the 'people, plant and process' (the three 'Ps'). In terms of 'people', the competency and awareness of the factory teams of Salmonella risks and management requirements is critical, and adequate training and management systems are key resources. For 'plant', the focus is on designing and controlling the manufacturing environment to ensure good manufacturing practices through sanitation approaches such as process and equipment design, segregation of microbiological hot and cold zones, airflow and condensation management, and overall maintenance to keep the facility in a 'fit for purpose' state. For 'process', there is a need for ongoing evaluation of the manufacturing systems to ensure proper critical control point identification, validation that the systems can provide the level of control needed, monitoring and verification that that control is being delivered, and review of material and personnel flows, sanitation programmes, prevention of cross-contamination, water control efforts, and the overall quality and food safety management process.

The three 'Ps' form the foundation for managing Salmonella (and other microbiological risks). Failure to have all in place would increase the risk of potential Salmonella contamination issues. In addition to the three 'Ps', it is critical to implement a verification programme that is statistical in design, sensitive and robust. This involves sampling the process environment, raw and in-process materials and finished products, including performing microbiological tests to verify the effectiveness of the control programmes. The selection of sampling locations, frequencies, quantities and test $\operatorname{method}(\mathrm{s})$ is also critical to the sensitivity and reliability of the programme. Again, a key to implementation of a successful verification programme is appropriate validation to ensure that verification system is capable of detecting potential issues before they can have an impact on product safety. An integral part of a proactive approach to the microbiological safety of pet foods is establishment of 'escalation criteria', corrective actions and communication plans based on the microbiological data. This structured approach helps ensure a standardised means of addressing potential issues and to provide the necessary awareness to manage potential risks.

\section{Evaluation of novel ingredients and potential toxicants}

The growing demand for food (for humans and pets), as well as animal feed, has led to increasing use of unconventional foodstuffs. The determination of the safety of new food constituents, additives or contaminants is always a daunting challenge. This is particularly an issue when the chemicals of concern are novel ingredients that have not been previously used in foods or were produced using novel technologies. These challenges are even more daunting in pet foods where manufacturers must consider both the unique metabolism of individual species and the differences that exist among breeds within a species. This requires a thorough understanding of how comparative toxicology must be considered in evaluating novel ingredients, and how this has had an impact on recent events in regulations that have an impact on the issue.

There are two aspects of general toxicology particularly pertinent to this topic: species differences in sensitivity to chemicals and the use of in vitro technologies to screen novel compounds to predict in vivo effects. It is well documented in the veterinary pharmacology literature and embedded in animal health regulations that species differences exist relative to all aspects of chemical and drug processing by animals, from absorption to metabolism to elimination and inherent biological activity. The US National Research Council ${ }^{(11)}$ study on safety of dietary supplements in animals clearly states that safety in humans does not predict safety in pets (e.g. garlic, aspirin), suggesting that historic use in human food does not guarantee safety in pets. Similarly, safety in laboratory rodents does not equate to safety in pets, nor would widespread use in dog food assure safety in cats. There are many other factors unique to individual species nutrition and feeding practices that confound this issue.

Based on a similar logic, the human toxicology and risk assessment community is realising that relying on laboratory animal screening tests alone does not predict human effects. This has led to a paradigm shift, best embodied in the $\mathrm{NRC}^{(12)}$ report's concept of toxicity testing in the twenty-first century, which would first identify active principles and define mechanism of action to help select appropriate animal models for testing. Importantly, an animal model or experimental system useful for assessing absorption may not be relevant for assessing toxicity. A bank of model systems of different complexities linked by quantitative models is needed. A similar scheme could be developed for pets. However, relevant in vitro screens are not available and biological endpoints (i.e. adverse effects) in target species (e.g. dogs or cats) have not been well defined as they have in humans.

Pet food manufacturers are always looking for new sources of nutrients that can enhance the nutritional well-being of pets. This includes new energy sources, protein sources, micronutrients and dietary supplements that may be 'novel' ingredients. What are 'novel' ingredients? The comparative toxicology discussion above could define a novel ingredient as one which has not been used in the species of concern since use in another species does not assure safety. However, the more interesting case is a truly novel ingredient with unique chemistry which has never been used as a feed additive or supplement. This could be a chemical found in a new raw material or natural food source, or one synthetically produced using novel chemistry or biotechnology approaches. What is the best method to screen these compounds? A variation on this theme is a 'normal' chemical produced using 'novel' techniques including nanotechnology or GM organisms. What about nano-formulations of existing substances? There are situations where new research identifies 'novel' clinical syndromes, an excellent example being the association of Balkan endemic nephropathy with aristolochic 
acid from plant contaminants, rather than as historically believed from ochratoxin exposure due to mould ${ }^{(13,14)}$.

Finally, there are the recent cases of economic adulterants being introduced in the food supply (e.g. melamine/cyanuric acid). How can these compounds be detected using screening methods before a toxicological incident defines the endpoint? This was particularly difficult with melamine and cyanuric acid since, individually, the compounds are considered to have low toxicity and only have an adverse effect when fed in combination $^{(15)}$. Thus, traditional testing of pure compounds would not identify the synergistic toxicity of the two compounds when consumed simultaneously. In recent years, events such as globalisation of trade and global climate shifts have exacerbated many of these issues and greatly increased the number of 'novel' ingredients of all definitions. This issue is multifaceted since techniques to detect and assess safety must be developed, and, then, once an ingredient is determined to be of concern, screening methods instituted to ensure production of a safe food are sustainable in the face of regulatory issues.

Despite the wide scientific uncertainty associated with novel ingredients, the international regulatory environment is often incoherent and definitely not harmonised for pet food additives. Many issues exist in the classification of supplements $v$. additives $v$. functional foods $v$. contaminants and adulterants. The US FDA has recently issued guidelines (http://fda. gov/Food/FoodIngredienttsPackaging/GenerallyRecognizeda asSafeGRAS/default.htm and http://www.fda.gov/AnimalVete rinary/ResourcesforYou/ucm047111.htm) to clarify Generally Regarded As Safe status. Of great importance to the toxicology community are differences in regulatory philosophies concerning specific additives (e.g. hormones and GM organisms) and the use of animals to detect adverse effects. In Europe, there is a continuing movement to ban all animal testing and replace with in silico quantitative structure-activity relationship risk modelling (e.g. REACH). However, accurate quantitative structure-activity relationship models cannot be developed in the absence of sound biological data to define relevant endpoints. For example, the European Parliament in July proposed suspension of sale of all food containing ingredients derived from nanotechnology ${ }^{(16)}$.

The determination of the safety of novel ingredients in pet food is a complex issue, much as it is for human food. The difference is that we know much more about human sensitivity to chemical toxicity than we do for pets, and we have much greater resources in human food safety to define syndromes and even screen feed sources. In the pet food industry, where manufacturers are under economic pressure, quality of raw materials are often less than that used for human food, and in many cases, science has not identified whether a specific chemical is toxic under conditions of use for a specific species or breed. Uniform definitions of dose must be established and active ingredients identified. Studies conducted in target species for palatability or nutritional effect should be designed, conducted and documented so that results can be used in hazard identification analyses should adverse effects be detected. However, the greatest challenge is to identify sensitive in vitro and in silico computational techniques that can screen out potential toxicants with a reasonable degree of accuracy. Only in this fashion can this field move forward on the foundation of strong science.

\section{New tools for evaluating pet foods}

As concerns about the safety of pet foods has increased, there has been increasing emphasis on being able to rapidly and cost-effectively evaluate ingredients and finished products for the presence of an array of chemical and microbiological hazards. Current laboratory methods for the identification of chemical contaminants in food use the so-called 'target list' approach. A predefined list of compounds is detected by the employment of customised extraction and analysis methods. This approach is appropriate for the quantification of compounds at trace levels for routine monitoring purposes where the target compound is known, but is limited when an unanticipated contamination threat arises. In these circumstances, exhaustive deployment of targeted analysis methods is time-consuming, expensive and often unsuccessful. Thus, substantial research is now focused on the development of non-targeted detection methods that are able to rapidly determine the presence of unknown contaminants. By obtaining a detailed understanding of what is naturally and normally present in foods, it becomes possible to monitor the food chain for abnormalities. A profiling or screening approach thus facilitates the determination of a wide range of issues in the areas of food fraud and food safety. While holistic monitoring of food has been the 'holy grail' for a number of years, it is only in recent times that instrumentation technology has advanced to the point where this has become feasible.

A number of high-profile issues have highlighted the need for broader-ranging determination of the composition of foodstuffs. The recent adulteration of pet food and milk products with melamine described above is a good example of how traditional approaches can fail. The compound was added to give false readings of protein content. Reported levels in some products were as high as $6.6 \%$, but since melamine was not routinely measured in foods and ingredients, there was wide penetration of the supply chain. Other widely reported adulteration issues include the use of carcinogenic dyes (e.g. Sudan I) ${ }^{(17)}$ to enhance the colour of products such as spices, an adulteration designed to improve the saleability of goods by enhancing their appearance. Other issues such as the addition of protein from cows and pigs to chicken products pose ethical and religious questions to humans and present a safety risk in the animal feed sector due to the potential for the perpetuation of transmissible spongiform encephalopathies. All of these issues would have benefited from recent advances in non-targeted detection methodology, which may have helped to avoid costly product recalls.

The pet food community is increasingly turning to analytical methodologies such as NMR spectroscopy and high-resolution liquid chromatography MS. NMR spectra usually possess a unique combination of chemical properties such as J-couplings, chemical shifts, nuclear Overhauser enhancements and diffusion rates, potentially facilitating automated molecular 
characterisation $^{(18-20)}$. In recent years, rapid developments in instrument design have resulted in significant improvements to both resolution and sensitivity. The inverse relationship between sensitivity and measurement time leads to a compromise between the desired detection limit and the time taken to acquire the NMR spectrum. Recent improvements in NMR sensitivity can therefore be used to obtain more rapid measurements and thus improve sample throughput. In order to extract key information from the NMR spectra of complex mixtures, a range of chemometric techniques have been used in combination with NMR spectroscopic data to, for example, detect characteristic differences in the chemical composition of foodstuffs ${ }^{(21)}$. This approach identifies deviations from normality in relation to product and ingredient composition. Once detected, an abnormal sample can be scrutinised using a range of NMR techniques to determine the nature of the problem.

There is complementarity between NMR spectroscopy and high-resolution liquid chromatography MS. The main benefits of using this combined approach are the relatively easy sample preparation strategy and short analysis times. Very high mass accuracy from the MS complements the highly specific data generated by NMR spectroscopy. Analysis of a variety of contaminants in relatively dirty extracts at very low concentrations can be achieved. The structure elucidation power of the NMR is then used to categorically determine the nature of an unexpected contamination incident. Using this approach, the detection of small quantities of unknown compounds in complex mixtures has been demonstrated without a priori knowledge of potential contaminants.

An increasingly global and complex supply chain associated with pet foods further complicates the already substantial challenge of assuring pet food safety. At the same time, new technologies continually emerge that can have an impact on every link of the supply chain, from farm to factory to food bowl. In this regard, several areas stand out as being ones to watch. Supply chain monitoring, decreasing costs and increased sophistication for bar code, radio frequency identification, wireless communication and global positioning satellite technologies make it feasible to trace the path of food ingredients and products throughout their life $\mathrm{e}^{(22-31)}$. On top of the basic when-and-where chronology, it is possible to gather additional data (metadata) through sensor networks ${ }^{(32)}$. For example, temperature, light, humidity, oxygen concentration or output from specialised analytical instruments can be monitored at relevant points. A simple implementation involves the use of smart packaging that can provide an instant visual check for mishandling or adverse conditions ${ }^{(26,33)}$. Information of this nature can be of great value for both realtime decisions and retrospective analysis of problems.

With the complexity of the supply chain, geographic information systems and data analytics are likely to play an increasingly important role in understanding and managing risk. For example, if we know the origin of a given ingredient, what else do we know about the local conditions at the time it originated? One thing we can easily find out about is weather. Was it unusually hot, cool, wet or dry? Are the weather conditions associated with threats to food such as fungi or pests? Another thing we might want to know about is regionally specific problems and threats. Had there been any recent outbreaks of disease in people, pets or livestock ${ }^{(34-37)}$ ? Are there known, localised biological threats, such as the toxic plant Aristolochia clematitis in the Balkans? Are conditions especially ripe for economic adulterers, criminals or terrorists? Taken together, these types of metadata constitute intelligence. When the food supply was local, this intelligence was relatively easy to gather and share in a community. With a global food supply, it is much more difficult for food manufacturers to keep tabs on what is happening in far-flung regions of the world. Geographic information system technologies can help manage the relevant data and, with the assistance of informatics and analytics, build local intelligence on a global scale.

Foods and food ingredients are notoriously difficult to analyse. They are complex, inconsistent and unstable. Further, they are physically heterogeneous, with multiple phases (such as fat, water and solids) that can sequester organic compounds, microbes and foreign material. As discussed above, the recovery of compounds of interest from this complex matrix has traditionally involved tedious sample preparation, extraction and purification. New technologies that allow for direct analysis of food materials with little, if any, sample preparation are greatly needed, and real progress is being made on this front. An example is new surface-sampling MS techniques that bypass the traditional extraction methods yet still provide high sensitivity and specificity ${ }^{(38)}$. Some useful analyses can now be performed in stand-off mode, where no direct contact with food materials is required ${ }^{(39)}$. Another trend is the steady miniaturisation and automation of analyses through microfluidic or lab-on-a-chip technologies ${ }^{(40-46)}$. These trends work synergistically, for as samples get smaller, and their preparation gets simpler, automation becomes more feasible. The ultimate outcome will be faster routine analyses that require less skilled labour and deliver greater return on capital investment.

As prevention of food safety concerns is increasingly being pushed back to the farm or the country of origin ${ }^{(47)}$, the ability to bring analytical capability to the field to make decisions about ingredients is increasingly viewed as the way to avoid the unnecessary shipment of food ingredients that will not meet the pet food manufacturer's specification or the government's regulations. This trend is driving the demand for fielddeployable analytical technologies. Instrumentation and tests that can be used by farmers, truck drivers, factory workers and government inspectors to make quick decisions are of particular value. In terms of analytical instrumentation, two examples of versatile technologies that have been successfully miniaturised are $\mathrm{MS}^{(48,49)}$ and Raman spectroscopy ${ }^{(50-54)}$. Both of these are powerful methods for analysing mixtures and have great potential for the food industry. Genomic technologies are also advancing at a rapid pace and will certainly lead to new field tests, as well as field-sampling procedures to be accompanied by laboratory analysis. In many cases, development of field-deployable devices and tests has been driven by law enforcement, defence and security needs ${ }^{(55)}$. Some of the products on the market can be used directly 
or adapted for food safety, but purpose-specific products will also be needed. Cooperation between technology developers, device manufacturers, food manufacturers and government will be required to accelerate the deployment of new tools for food safety.

All told, widely dispersed, inexpensive and easy-to-use technologies can be powerful tools for advancing pet food safety. Their proliferation can thus be of great benefit, but it also presents big challenges: how to stay current on a large, rapidly growing set of technologies; how to perform costbenefit analyses quickly and effectively; how to manage a swelling body of data and extract useful information from it; how to allocate scarce resources in selecting the technologies to adopt.

In summary, recent events have demonstrated that pet foods can be the vehicle for hazards that can adversely affect pets and their owners. Thus, pet food safety is an emerging concern of high interest to consumers. The pet food industry is rapidly responding by mobilising enhanced management systems and calling on the scientific community to find an effective means to mitigating risks. Such an effort is critical to maintaining consumer confidence in a class of products that has contributed profoundly to the health and well-being of our companion animals.

\section{Acknowledgements}

The authors would like to thank MARS Petcare and its Waltham Centre for Pet Nutrition for its support in allowing the authors to participate in the 2010 Waltham Symposium. The authors' attendance at the Waltham Symposium was supported by MARS, Inc. Additionally, they are external and/or internal members of the Food Safety Subcommittee of the MARS Scientific Advisory Committee. R. L. B. was responsible for the section on the epidemiology of foodborne diseases associated with pet foods. R. C. B. was responsible for the section on changes being implemented by the pet food industry to increase microbiological safety. J. E. R. was responsible for the section on the evaluation of novel ingredients. A. J. C. and R. S. were jointly responsible for the section on new tools for evaluating pet foods.

\section{References}

1. Food and Drug Administration (FDA) (2007) Melamine pet food recall of 2007. http://www.fda.gov/AnimalVeterinary/ SafetyHealth/RecallsWithdrawals/ucm129575.htm (accessed 6 January 2011).

2. World Health Organization (2009) Toxicological and Health Aspects of Melamine and Cyanuric Acid. Geneva: WHO.

3. Food and Drug Administration (FDA) (2005) Diamond pet food recalled due to aflatoxin. http://www.fda.gov/Safety/ Recalls/ArchiveRecalls/2005/ucm111929.htm （accessed 6 January 2011).

4. Galton MM, Scatterday JE \& Hardy AV (1952) Salmonellosis in dogs I: bacteriological, epidemiological and clinical considerations. J Infec Dis 91, 1-5.

5. Mackel DC, Galton MM, Gray H, et al. (1952) Salmonellosis in dogs IV: prevalence in normal dogs and their contacts. $J$ Infect Dis 91, 15-18.
6. Day WH, James E \& Heather CD (1963) Salmonellosis in the dog. Am J Vet Res 24, 156-157.

7. Morse EV \& Duncan MA (1975) Canine salmonellosis: prevalence, epizootiology, signs and public health significance. J Am Vet Med Assoc 167, 817-820.

8. LeJeune JT \& Hancock DD (2001) Public health concerns associated with feeding raw meat diets to dogs. J Am Vet Med Assoc 219, 1222-1225.

9. Centers for Disease Control and Prevention (CDC) (2006) Human salmonellosis associated with animal-derived pet treats - United States and Canada, 2005. MMWR Morb Mortal Wkly Rep 55, 702-705.

10. Centers for Disease Control and Prevention (CDC) (2008) Multistate outbreak of human salmonellosis infections caused by contaminated dry dog food - United States, 2006-2007. MMWR Morb Mortal Wkly Rep 57, 521-524.

11. National Research Council (NRC) (2008) Safety of Dietary Supplements for Horses, Cats, and Dogs. Washington, DC: National Academies Press.

12. National Research Council (NRC) (2007) Toxicity Testing in the 21st Century: A Vision and a Strategy. Washington, DC: National Academies Press.

13. Debelle FD, Vanherweghem J-L \& Nortier JL (2008) Aristolochic acid nephropathy: a worldwide problem. Kidney Int 74, 158-169.

14. Jankovic Velickovic L, Takanori H \& Stefanovic V (2009) Molecular markers in upper urothelial carcinoma associated with Balkan endemic nephropathy. Aristolochic acid as the major risk factor of the worldwide disease. Scientific World $J \mathbf{9}$, $1360-1373$.

15. Baynes RE \& Riviere JE (2010) Risks associated with melamine and related triazines contamination of food. Environ Health Threat J 3, e5, 1-10.

16. European Parliament (2011) Press release: nanotechnology. http://www.europarl.europa.eu/en/headlines/content/20110 324STO16430/html/EU-countries-reject-EP-call-for-labellingof-clone-derived-food (accessed 6 January 2011).

17. Stiborova M, Martinek V, Rydlova H, et al. (2002) Sudan I is a potential carcinogen in humans - evidence for its metabolic activation and detoxication by human recombinant cytochrome P450 1A1 and liver microsomes I. Cancer Res $\mathbf{6 2}$, $5678-5684$.

18. Charlton AJ, Donarski JD, Jones SA, et al. (2006) The development of cryoprobe nuclear magnetic resonance spectroscopy for the rapid detection of organic contaminants in potable water. J Environ Monitor 8, 1106-1110.

19. Charlton AJ, Robb P, Donarski JA, et al. (2008) Non-targeted detection of chemical contamination in carbonated soft drinks using NMR spectroscopy, variable selection and chemometrics. Anal Chim Acta 618, 196-203.

20. Charlton AJ, Donarski JA, May BD, et al. (2009) Optimisation of NMR methodology for non-targeted detection of water contaminants. In Water Contamination Emergencies: Collective Responsibility, [J Gray and KC Thompson, editors]. Cambridge: RSC Publishing.

21. Charlton AJ, Wrobel MS, Stanimirova I, et al. (2010) Multivariate discrimination of wines with respect to their grape varieties and vintages. Eur Food Res Technol 231, 733-743.

22. Brody AL, Bugusu B, Han JH, et al. (2008) Innovative food packaging solutions. J Food Sci 73, R107-R116.

23. Kumar P, Reinitz HW, Simunovic J, et al. (2009) Overview of RFID technology and its applications in the food industry. J Food Sci 74, R101-R106.

24. Ruiz-Garcia L, Lunadei L, Barreiro P, et al. (2009) A review of wireless sensor technologies and applications in agriculture 
and food industry: state of the art and current trends. Sensors 9, $4728-4750$

25. Wang N, Zhang NQ \& Wang MH (2006) Wireless sensors in agriculture and food industry - recent development and future perspective. Comp Electron Agric 50, 1-14.

26. Yam KL, Takhistov PT \& Miltz J (2005) Intelligent packaging: concepts and applications. I Food Sci 70, R1-R10.

27. Gebresenbet G, Wikner I, van der Water G, et al. (2003) A smart system for surveillance of animal welfare during transport. Dtsch Tieraerztl Wochenschr 110, 494-498.

28. Li H, Chen XL, Cai XB, et al. (2005) Assessment of soil quality using GIS \& RS. In IGARSS 2005: IEEE International Geoscience and Remote Sensing Symposium, vols 1-8, Proceedings, pp. 2972-2975.

29. Tibola CS, Fachinellol JC, Rombaldi CV, et al. (2008) Traceability of peaches from integrated production in south Brazil. Sci Agric (Piracicaba, Braz) 65, 10-15.

30. Whittier JC, Shadduck JA \& Golden BL (2003) Secure identification, source verification of livestock - the value of retinal images and GPS. In Precision Livestock Farming, pp. 167-172 [S Cox, editor]. Wageningen, NL: Wageningen Academic Publishers.

31. Zhang J, Liu L, Mu WS, et al. (2009) Development of temperature-managed traceability system for frozen and chilled food during storage and transportation. J Food Agric Environ 7, 28-31.

32. Gorman BL, Resseguie DR \& Tomkins-Tinch C (2009) Sensorpedia: information sharing across incompatible sensor systems. In 2009 International Symposium on Collaborative Technologies and Systems, pp. 448-454.

33. Mahalik NP \& Nambiar AN (2010) Trends in food packaging and manufacturing systems and technology. Trends Food Sci Technol 21, 117-128.

34. Fuchs K, Wagner P \& Kofer J (2001) VETGIS((R))-Styria - a geographic information system as a tool for epidemiological research for the veterinary administration. Wien Tieraerztl Monatsschr 88, 246-251.

35. Kofer J, Kleb U \& Pless P (2006) The Styrian Salmonella Monitoring Programme for pork production. $J$ Vet Med, Ser B 53, 209-212.

36. Jepsen MR, Fisher I, Galle M, et al. (2008) Creating an online atlas of Salmonella serotypes in Europe. Eurosurveillance 13, $1-3$.

37. Riolo F (2009) A web-based GIS for zoonoses monitoring. ESRI Map Book Online 24, http://www.esri.com/mapmus eum/mapbook_gallery/volume24/health1.html.

38. van Berkel GJ, Pasilis SP \& Ovchinnikova O (2008) Established and emerging atmospheric pressure surface sampling/ionization techniques for mass spectrometry. J Mass Spectrom 43, 1161-1180.
39. van Neste CW, Senesac LR \& Thundat T (2009) Standoff spectroscopy of surface adsorbed chemicals. Anal Chem 81, 1952-1956.

40. Escarpa A, Gonzalez MC, Gil MAL, et al. (2008) Microchips for CE: breakthroughs in real-world food analysis. Electrophoresis 29, 4852-4861.

41. Palchetti I \& Mascini M (2008) Electroanalytical biosensors and their potential for food pathogen and toxin detection. Anal Bioanal Chem 391, 455-471.

42. Skurtys O \& Aguilera JM (2008) Applications of microfluidic devices in food engineering. Food Biophys 3, 1-15.

43. Lauri A \& Mariani PO (2009) Potentials and limitations of molecular diagnostic methods in food safety. Genes Nutr $\mathbf{4}$, $1-12$.

44. Sozer N \& Kokini JL (2009) Nanotechnology and its applications in the food sector. Trends Biotechnol 27, 82-89.

45. Shin GW, Hwang HS, Chung B, et al. (2010) Recent developments in CE-based detection methods for food-borne pathogens. Electrophoresis 31, 2137-2153.

46. Neethirajan S, Kobayashi I, Nakajima M, et al. (2011) Microfluidics for food, agriculture and biosystems industries. $L a b$ Chip 11, 1574-1586.

47. Waterman FA \& Ibrahim JK (2009) Foodborne outbreaks and agricultural practices: should public health prevention start in the field. $J$ Environ Health 71, 61-63.

48. Hart KJ, Wise MB, Griest WH, et al. (2000) Design, development, and performance of a fieldable chemical and biological agent detector. Field Anal Chem Technol 4, 93-110.

49. Xu W, Manicke NE, Cooks GR, et al. (2010) Miniaturization of mass spectrometry analysis systems. JALA 15, 433-439.

50. Jordan G, Thomasius R, Schroder H, et al. (2009) Non-invasive mobile monitoring of meat quality. $J$ Verbraucherschutz Lebensmittelsicherh 4, 7-14.

51. Cheng Y, Dong YY, Wu JH, et al. (2010) Screening melamine adulterant in milk powder with laser Raman spectrometry. J Food Compos Anal 23, 199-202.

52. Cheung W, Shadi IT, Xu Y, et al. (2010) Quantitative analysis of the banned food dye Sudan-1 using surface enhanced Raman scattering with multivariate chemometrics. J Phys Chem C 114, 7285-7290.

53. Sowoidnich K, Schmidt H, Maiwald M, et al. (2010) Application of diode-laser Raman spectroscopy for in situ investigation of meat spoilage. Food Bioprocess Technol 3, 878-882.

54. Gu C, Yang XA, Zhang J, et al. (2010) Fiber sensors for molecular detection. PrOC SPIE 7851, 785105-1-785105-14.

55. Izake EL (2010) Forensic and homeland security applications of modern portable Raman spectroscopy. Forensic Sci Int 202, 1-8. 